\title{
Asteroid Crewed Segment Mission Lean Development
}

\author{
Joe Gard, Mark McDonald, Wayne Jermstad \\ NASA Johnson Space Center, Houston, TX 77058
}

The next generation of human spaceflight missions presents numerous challenges to designers that must be addressed to produce a feasible concept. The specific challenges of designing an exploration mission utilizing the Space Launch System and the Orion spacecraft to carry astronauts beyond earth orbit to explore an asteroid stored in a distant retrograde orbit around the moon will be addressed. Mission designers must carefully balance competing constraints including cost, schedule, risk, and numerous spacecraft performance metrics including launch mass, nominal landed mass, abort landed mass, mission duration, consumable limits and many others. The Asteroid Redirect Crewed Mission will be described along with results from the concurrent mission design trades that led to its formulation. While the trades presented are specific to this mission, the integrated process is applicable to any potential future mission.

The following trades were critical in the mission formulation and will be described in detail: 1) crew size, 2) mission duration, 3) trajectory design, 4) docking vs grapple, 5) extravehicular activity tasks, 6) launch mass and integrated vehicle performance, 7) contingency performance, 8) crew consumables including food, clothing, oxygen, nitrogen and water, and 9) mission risk.

The additional Orion functionality required to perform the Asteroid Redirect Crewed Mission and how it is incorporated while minimizing cost, schedule and mass impacts will be identified. Existing investments in the NASA technology portfolio were leveraged to provide the added functionality that will be beneficial to future exploration missions. Mission kits are utilized to augment Orion with the necessary functionality without introducing costly new requirements to the mature Orion spacecraft design effort. The Asteroid Redirect Crewed Mission provides an exciting early mission for the Orion and SLS while providing a stepping stone to even more ambitious missions in the future. 


\title{
Asteroid Crewed Segment Mission Lean Development
}

\author{
Joe A. Gard ${ }^{1}$ and Mark Mcdonald. ${ }^{2}$ \\ NASA Johnson Space Center, Houston, TX 77058 \\ Wayne Jermstad \\ NASA Johnson Space Center, Houston, TX 77058
}

The next generation of human spaceflight missions presents numerous challenges to designers that must be addressed to produce a feasible concept. The specific challenges of designing an exploration mission utilizing the Space Launch System and the Orion spacecraft to carry astronauts beyond earth orbit to explore an asteroid stored in a distant retrograde orbit around the moon will be addressed. Mission designers must carefully balance competing constraints including cost, schedule, risk, and numerous spacecraft performance metrics including launch mass, nominal landed mass, abort landed mass, mission duration, consumable limits and many others. The Asteroid Redirect Crewed Mission will be described along with results from the concurrent mission design trades that led to its formulation. While the trades presented are specific to this mission, the integrated process is applicable to any potential future mission.

Critical trades in the mission formulation include: crew size, mission duration and trajectory design, docking vs. grapple, launch mass performance, and crew consumables including food, clothing, oxygen, nitrogen and water, and mission risk. The additional Orion functionality required to perform the Asteroid Redirect Crewed Mission and how it is incorporated while minimizing cost, schedule and mass impacts will be identified. Existing investments in the NASA technology portfolio were leveraged to provide the added functionality that will be beneficial to future exploration missions. Mission kits are utilized to augment Orion with the necessary functionality without introducing costly new requirements to the mature Orion spacecraft design effort. The Asteroid Redirect Crewed Mission provides an exciting early mission for the Orion and SLS while providing a stepping-stone to even more ambitious missions in the future.

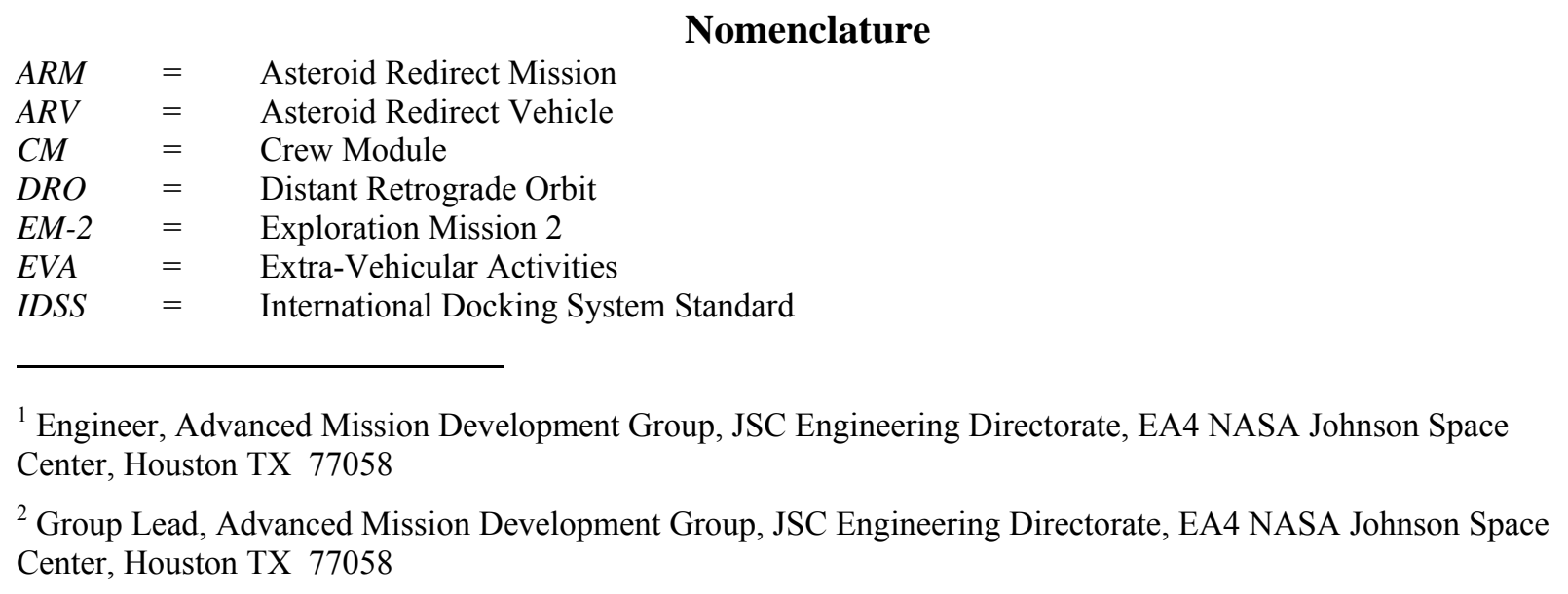




$\begin{array}{lll}\text { LAS } & = & \text { Launch Abort System } \\ \text { LEO } & = & \text { Low-Earth Orbit } \\ \text { LOCI } & = & \text { Laser Optical Camera Instrument } \\ \text { MACES } & = & \text { Modified Advanced Crew Escape Suit } \\ \text { NORS } & = & \text { Nitrogen Oxygen Resupply System } \\ P C S & = & \text { Pressure Control system } \\ \text { PLS } & = & \text { Primary Life Support System } \\ \text { RPOD } & = & \text { Rendezvous, Proximity Operations and Docking } \\ \text { SA } & = & \text { Spacecraft Adapter } \\ \text { SLS } & = & \text { Space Launch System } \\ \text { SM } & = & \text { Service Module } \\ \text { SOP } & = & \text { Secondary Oxygen Pack } \\ \text { STORM } & = & \text { Sensor Test for Orion RelNav Risk Mitigation } \\ T L I & = & \text { Trans Lunar Injection } \\ T M G & = & \text { Thermal Micrometeoroid Garment }\end{array}$

\section{INTRODUCTION}

NASA is currently building a new human spacecraft, Orion, and rocket system, Space Launch System (SLS), that will facilitate a new era of space exploration. A series of increasingly challenging missions awaits, and this new spacecraft will take us farther than we've gone before. It will be most advanced spacecraft ever built capable enough to take us to a variety of destinations including exploring a captured near earth asteroid. Under the Asteroid Redirect Mission (ARM) plan, a near earth asteroid would be redirected into a Distant Retrograde Orbit (DRO) around the Earths Moon where an Orion capsule launched atop an SLS rocket will carry crew members and the equipment necessary to explore the asteroid, collect samples and return safely to Earth.

Planning a mission to explore the asteroid poses several challenges to mission designers including balancing competing constraints including cost, risk and spacecraft performance metrics like launch mass, mission duration and consumable limitations. In order to demonstrate the feasibility of a crewed asteroid mission many critical parameters including mission objectives, crew size, mission duration, crew consumables and spacecraft performance are examined to develop a feasible mission plan.

\section{ARCM MISSION OVERVIEW}

\section{A. Reference Mission Schedule}

Current NASA policy is to perform asteroid missions as soon as 2021 and before 2025. For planning purposes asteroid 2009BD was selected as a feasible target. The reference ARCM evaluated for this study targets Asteroid Redirect Vehicle (ARV) rendezvous with 2009BD and redirecting the asteroid to a DRO no earlier than November 2024. However performance assessment showed than an Orion rendezvous and dock with the ARV is feasible in May 2024 prior to full DRO insertion by allowing the ARV to coast along the 'spiral' during the short docked operations. Once the Orion has undocked, the ARV can continue to the final DRO.

\section{B. Asteroid Redirect Crewed Mission Concept Overview}

After capturing the asteroid, the ARV spacecraft is used to redirect the trajectory of an asteroid, capture it into the Earth-Moon system, and transfer it to a stable DRO with an orbit altitude of roughly $70,000 \mathrm{~km}$.

The crew will be transported to the DRO utilizing the Orion spacecraft launched atop a SLS rocket. The ARCM design reference mission is designed around the translunar transit, rendezvous and docking with the ARV in the DRO orbit and return to earth.

At the asteroid, crewmembers will perform two four-hour Extra-Vehicular Activities (EVA) to explore the asteroid and retrieve samples for Earth return. The ARCM design reference mission supports five days total time at the DRO for joint operations, with a day between EVAs and protection for a contingency day. Upon completion of 
integrated operations the Orion spacecraft returns to earth on a target line trajectory splashing down in the Pacific Ocean off the coast of California. The total mission duration is between 24 and 30 days depending on the specific epoch selected.

\section{Orion ARCM Reference Configuration}

The Orion spacecraft is a pressurized, crewed element that transports up to four crew members to space and returns them safely back to the Earth's surface at the end of a mission. The Orion provides all services necessary to support four crewmembers for up to 21 days. Figure 1 depicts a representative Orion configuration including the Crew Module (CM), Service Module (SM), Spacecraft Adapter (SA) and a Launch Abort System (LAS).

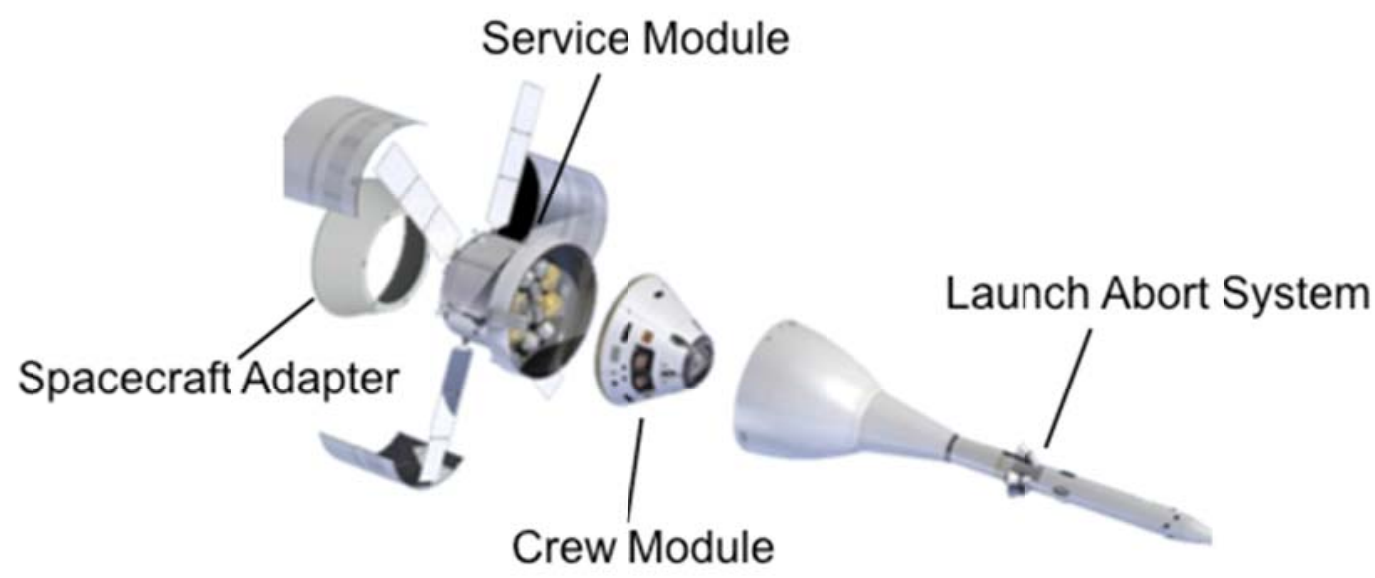

Figure 1: Orion Configuration

For this feasibility study, the Orion configuration is based on the Orion for the Exploration Mission-2 (EM-2) Lunar Orbit mission. The Orion vehicle configuration summary for the EM-2 mission is shown in Table 1 and includes provisions for four crewmembers for up to 21 days. For this study, the Orion reference configuration was augmented to add rendezvous and docking, EVA, and sample return functionality required to accomplish the ARCM objectives. To minimize the integrated Orion hardware and software configuration changes, the additional ARCM functionality is provided as add-on mission kits that will interface with the existing Orion systems where practical.

\section{Table 1: Orion EM-2 Reference Configuration}

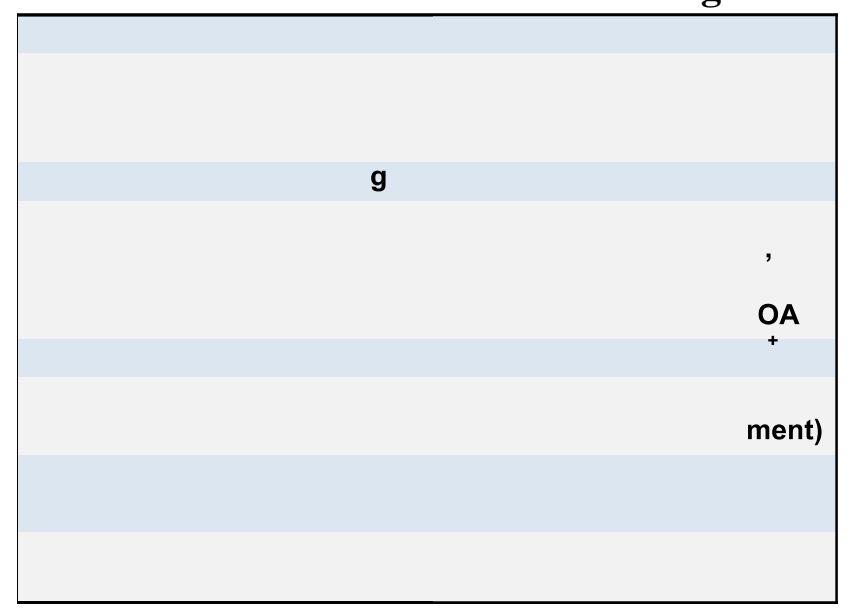


Table 2 contains a list of the ARCM kits required to support rendezvous and docking, EVA, and sample return objectives. The EVA Suit Kit contains the elements necessary to convert the launch and entry suit into an EVAcompatible suit. The EVA Servicing Kit contains hardware to replenish and recharge suit consumables between EVAs and provide communications between the ground and crew during the EVAs. The EVA Repress Kit provides cabin air required to repress the Orion cabin following the two EVAs. The EVA kits are designed to leverage existing development work or to utilize existing technology.

Table 2: Orion ARCM Functionality Kits

\begin{tabular}{|l|l|}
\hline Functionality & Kit \\
\hline EVA & EVA Suit Kit \\
& EVA Servicing Kit \\
& EVA Repress Kit \\
\hline Vehicle Mating & Relative Navigation Kit \\
& Docking Kit \\
\hline
\end{tabular}

The vehicle mating kits consist of: Relative Navigation Kit which includes the sensors required for rendezvous and proximity operations and a Docking Kit which includes the International Docking System Standard (IDSS) compliant mechanisms necessary to securely attach the two spacecraft. Although not included in the EM-2 mission, the Orion spacecraft is designed to accommodate the IDSS compliant mechanism without design modifications. The sensor data generated by the Relative Navigation Sensor kit during rendezvous and docking must be integrated into the Orion Guidance and Navigation software. Because the reference EM-2 mission does not include docking activities, the integration of the rendezvous sensors and guidance algorithms required for docking is not included in the EM-2 baseline.

\section{MISSION DESIGN TRADES}

\section{A. Trajectory}

The reference ARCM trajectories evaluated for this study targets an Orion launch to rendezvous and dock with the ARV/asteroid in a retrograde orbit around the moon. The trajectory utilizes powered lunar gravity assist maneuvers to assist the Orion spacecraft to and from the DRO. Several different trajectory approaches were examined including minimizing total duration, minimizing the number of burns required to perform the mission and minimizing the Orion $\Delta \mathrm{V}$ required to complete the mission.

The reference trajectory was selected based on minimizing the Orion $\Delta \mathrm{V}$ required in order to maintain some measure of abort capability. The reference trajectory is shown in Figure 2. The outbound leg requires 7 days with 1 day to rendezvous and dock with the ARV, 5 days of operations at the asteroid and 10 days to return to earth for a total mission duration of 23 days. 


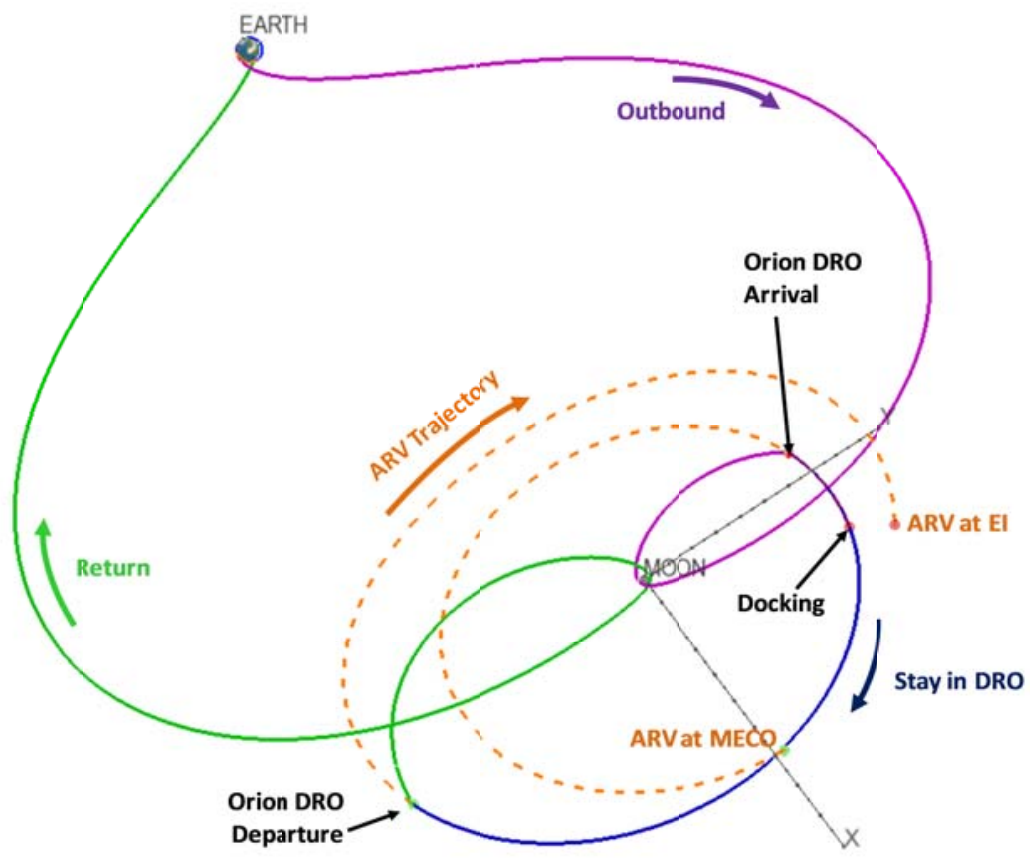

Figure 2 ARCM Reference Trajectory

\section{B. Orion Crew Mass}

As noted above, the Orion spacecraft is designed to accommodate 4 crewmembers for up to 21 days in space. In order to minimize the impact to the Orion system performance, a philosophy of minimizing the increase in vehicle mass was adopted. This required removing items from the Orion spacecraft to create a mass wedge that could be used to add the required ARCM mission kits and increase the duration. A review of the Orion systems showed that reducing the size of the crew from 4 to 2 could accommodate most of the additional mass.

In addition to the mass of two crew members, the mass of support equipment including: two launch and entry suits, two seats and 24 days worth of food and crew preference items were removed. Table 3 enumerates a tally of the items removed. Initially excess water, oxygen and nitrogen contained by the Service Module were also removed, however further analysis showed that maintaining a full compliment of SM consumables provided mission duration margin (up to 30 days) while still being able to meet Orion performance objectives. A detailed analysis of crew consumables is presented later in this paper.

Table 3: Orion Off-Loaded Mass

\begin{tabular}{|c|c|c|c|}
\hline & Orion Launch Mass for ARUM & Ib & $\mathrm{kg}$ \\
\hline \multirow{7}{*}{ 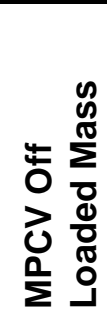 } & Crew & -360.0 & -163.3 \\
\hline & Crew Seat & -130.1 & -59.0 \\
\hline & GFE Crew Systems & -102.0 & -46.2 \\
\hline & Suits & -142.3 & -64. \\
\hline & SM Water* & 0.0 & 0. \\
\hline & SM Oxygen* & 0.0 & 0 . \\
\hline & SM Nitrogen ${ }^{\star \star}$ & 0.0 & \\
\hline
\end{tabular}

\section{EVA Kits}

The EVA kits provide EVA capabilities without modification to the baseline Orion vehicle design. The kits include: Suit Kit, Servicing Kit, Tool Kit, Sample Container Kit, Communications Kit, and Repress Kit. 


\section{III.C.1 EVA Suit Kit}

Before conducting a space walk, astronauts must put on a suit capable of protecting them from the harsh environment of space. For the last 30 years this has meant using the Extravehicular Mobility Unit (EMU) designed in the late 70s to support up to 8 hour EVAs. Unfortunately this suit is heavy and bulky and not well suited for a mass constrained mission such as ARCM. Even the next generation of space suits currently being designed by NASA would add too much mass to the mission.

In order to minimize the mass impact, and leverage existing hardware, the ARCM mission will be based on the current configuration launch and entry suit for Orion defined as the Baseline MACES. The suit is nominally worn during launch and entry operations, however with some modifications could be converted into a suit capable of supporting limited EVA operations.

The Baseline MACES modified to improve mobility for EVA operations, called the Enhanced MACES will be the launch and entry suit for the ARCM. The Enhanced MACES can be further configured to support the two EVAs during the ARCM is called the Primary Life Support System MACES (PLSS MACES). Using the launch and entry suit for EVAs is not without precedent. Gemini EVA experience demonstrated that with mobility enhancements, an EVA in a launch and entry suit is feasible. For the ARCM, the mass impact of using a dedicated EVA suit, similar to Shuttle, was too large to be accommodated and utilizing the same core suit for launch and entry and EVA saves nearly $100 \mathrm{~kg}$ of mass required to support EVAs.

Modifications to the Enhanced MACES will include the addition of arm bearings, flexible elbows, foot restraint compliant boots, sunshield-modified helmets, and additional pressure regulation valves as shown in Figure 3 . These design changes will be tested to quantify improvements above baseline capability for incorporation into the Enhanced MACES flight configuration.

The PLSS MACES configuration is created by integrating the Exploration Portable Life Support System (PLSS), Display and Control Module (DCM), Thermal Micrometeoroid Garment (TMG), associated support harnesses, and EVA gloves to the Enhanced MACES. These components comprise the EVA Suit Kit, which is an addition to the Orion baseline mass allocation.
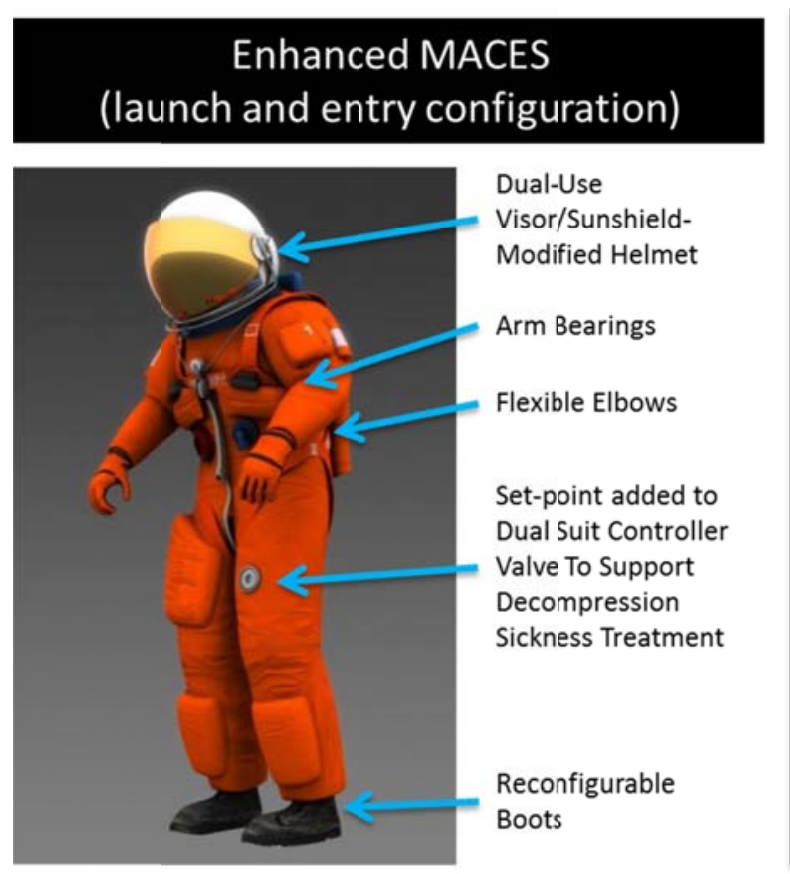
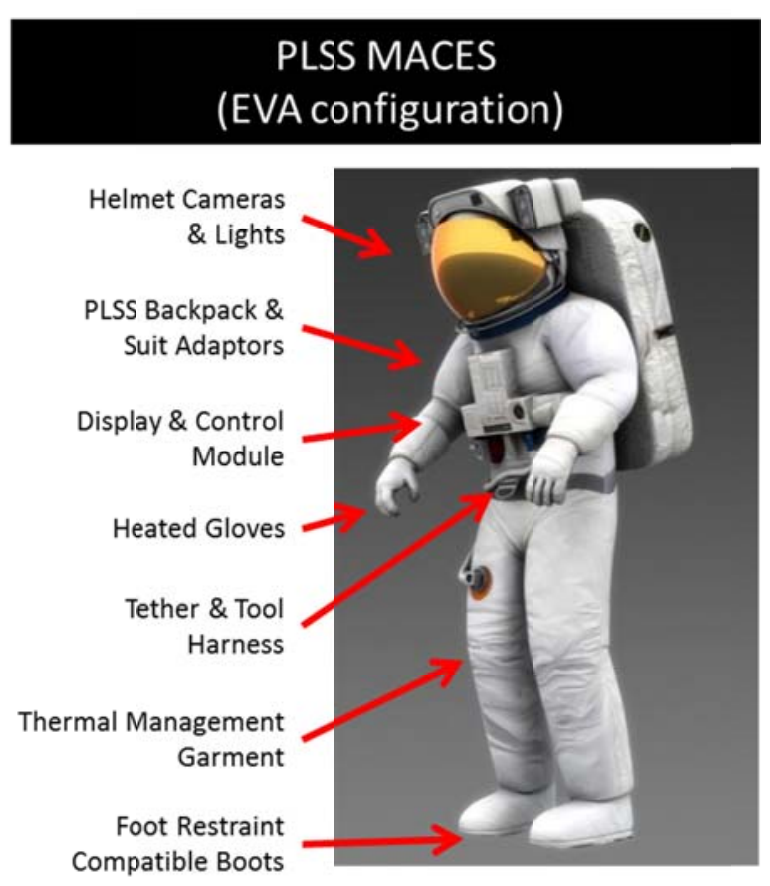

Figure 3 Conceptual Enhanced MACES and PLSS MACES 


\section{III.C.2 Exploration Portable Life Support System (PLSS)}

To provide the crew the necessary Oxygen and water during their spacewalk, two methods of support were evaluated. Long EVA umbilicals that could connect to the existing Orion high-pressure $\mathrm{O} 2$ system and a stand alone PLSS.

The geometry of the asteroid and ARV requires umbilicals of at least 100 ' in length which would significantly reduce the effectiveness of the EVA as one crew member would be responsible for ensuring the umbilical does not become ensnared on any equipment on the spacecraft or Asteroid. Additionally, umbilical based EVAs would use the Orion system oxygen to support the EVAs which would require larger Service Module Oxygen tanks, or supplemental oxygen tanks be stowed in the Orion cabin. The Orion life support is not designed to accommodate oxygen tanks in the crew cabin and an interface to utilize those tanks would have to be developed driving design changes to the Orion spacecraft.

The Exploration PLSS is an ongoing technology development project functioning independently of a specific suit for EVAs in a vacuum environment. The Exploration PLSS provides pressurization of the suit, oxygen for breathing, carbon dioxide removal, thermal control and communication. Use of a PLSS increases operational effectiveness because both crewmembers are available to support EVA related tasks.

\section{III.C.3 EVA Repress Kit}

The majority of the gases used for pressure control in the Orion are stored in tanks on the service module. Going from a 4-person crew to a 2-person crew for this mission will save oxygen, but the bulk of the gas needed for the repressurization is nitrogen, which is not substantially affected by crew size.

The EVA Repress Kit provides enriched air to fill the cabin after an EVA. The repress kit will be located within the Orion cabin allows for a crew-operated system minimizing the impact to existing Orion systems. Repress gas can be provided as separate oxygen and nitrogen or as mixed gas. However, manually mixing two gases requires precision measurements and operations in order to avoid creating a hazardous atmosphere in the cabin. Providing only nitrogen tanks in the cabin but using Orion oxygen would require integration of the automated system and manual operation. The Orion Pressure Control System (PCS) is not designed to accommodate constituent gas introduction into the cabin. While using separated nitrogen and oxygen is possible, this study judged that using a pre-mixed enriched air mixture is simpler. A pre-mixed enriched air repress system will maintain safe oxygen concentration, mitigating both the risk of fire and an unsafe low-oxygen environment. Based on cabin volume, this study assumes each repressurization will require $10.7 \mathrm{lbs}$. of oxygen and $25.3 \mathrm{lbs}$. of nitrogen. This oxygen/nitrogen ratio is slightly oxygen-poor to accommodate the release of pure oxygen into the cabin when the crew doffs the EVA suits.

The system concept includes a single tank with gas for both repressurizations, and valves and regulators. A single tank typically has a better surface area to volume ratio and requires only one set of interfaces, and thus would weigh less than two smaller tanks. Regulator and isolation valves were chosen to stop and start flow. A highpressure system will require at least two methods of stopping flow, and a regulator-valve system has approximately the same mass as two valves, but greatly simplifies operation because the crew doesn't have to stop the flow at exactly the right time to reach the target pressure. To save volume, this study assumes the tank is at 5000 Pisa internal pressure nominally, and a step-down regulator is used to reduce the pressure before delivery. The system would also have pressure gauges for visual inspection to verify that it does not leak and how much gas remains in the tank. Finally, a small mass placeholder for a frame or other attachment point was included in the mass estimates. The mass of the tank was based on a capacity to mass ratio developed from the ISS Nitrogen Oxygen Resupply System (NORS) tanks, which are designed to be portable and handled by the crew, much as this tank may be. The 
regulator and valve parts are based on mass estimates from Skylab EVA suit Secondary Oxygen Pack (SOP) system, which should be operable with an EVA gloved hand, and rated for high pressure.

\section{Docking Kit}

Docking and Grappling were evaluated as methods for attaching the Orion and ARV vehicles. Both methods have advantages and disadvantages that will be discussed in this section.

\section{III.D.1 Grappling}

Grappling the Orion and the ARV vehicles can be performed by installing a grapple fixture on the aft end of the ARV near the thrusters, and a grapple arm in the nose cone of the Orion. For this study, a deployable grapple arm based on existing articulating utilizing $3^{\text {rd }}$ generation harmonic drive joints was assessed. Orion will require minimal modifications to incorporate the grappling because the grapple arm can interface the spacecraft using existing structural ring docking interface. An independent RF hand controller is used to control the grapple arm so no integration with Orion flight software is required.

Preliminary analysis showed the worst case birthing box translation loads could be mitigated within 20 seconds after capture and provide a semi-rigid interface between the spacecraft. However, continuous active control of the grapple arm is required to maintain the distance between the vehicles and to withstand all loads. Mated loads between the vehicles must be minimized and changes to the integrated stack attitude may not be possible because of the loads induced into the grapple arm.

Grappling may provide lower mass impacts to the spacecraft than other approaches. The grapple arm estimated mass is $115 \mathrm{~kg}$ and the grapple fixture estimated mass is $36 \mathrm{~kg}$. However, the mass may increase if analysis shows the applied loads are higher than the arm can accommodate.

\section{III.D.2 Docking}

Unlike grappling, the docking mechanism does require higher integration with Orion systems including integration with the Orion flight software to command the NDS actuation and detect soft and hard capture modes. The mechanical interface to the Orion spacecraft is the structural ring connected to the docking interface.

The Docking mechanism has a higher mass impact than grappling with the active side weighing $341 \mathrm{~kg}$ and the passive mechanism weighing $114 \mathrm{~kg}$. However, because the docking mechanism provides an adequate structural load path, the docking mechanism can accommodate extended duration connections between spacecraft including attitude excursions using the spacecraft RCS jets. For extensibility, the docking mechanism provides data and power interfaces that can be mated without EVA reducing the number of inhibits that would be required for manual umbilical connections and simplifying the power design.

Traditionally, grappling has been used for transient coupling of spacecraft (i.e. satellite deployment and capture, ISS transition from rendezvous to birthed operations) but may not provide a long-term robust solution for attaching spacecraft. For long-term extensibility and utilization of the asteroid by commercial and international partners, Docking is the recommended method. For this study, the NASA Docking System (NDS) will be the docking interface for mating the Orion to the ARV.

\section{III.D.3 Docking Mechanism Placement}

The feasibility study examined several locations for the docking mechanism on the ARV including radially along the length of the ARV, and axially. The compact design of the ARV did not allow mounting the docking mechanism radially along the length because of array clearance and approach corridor constraints. Figure 3 shows the Orion docked to one of the considered ARV locations. Docking radially also causes potential issues because of the rates induced during docking.

Placing the docking mechanism axially allows for sufficient solar array clearance, maintains the preferred solar inertial attitude for both the ARV and Orion spacecraft and allows the greatest potential for extensibility. 

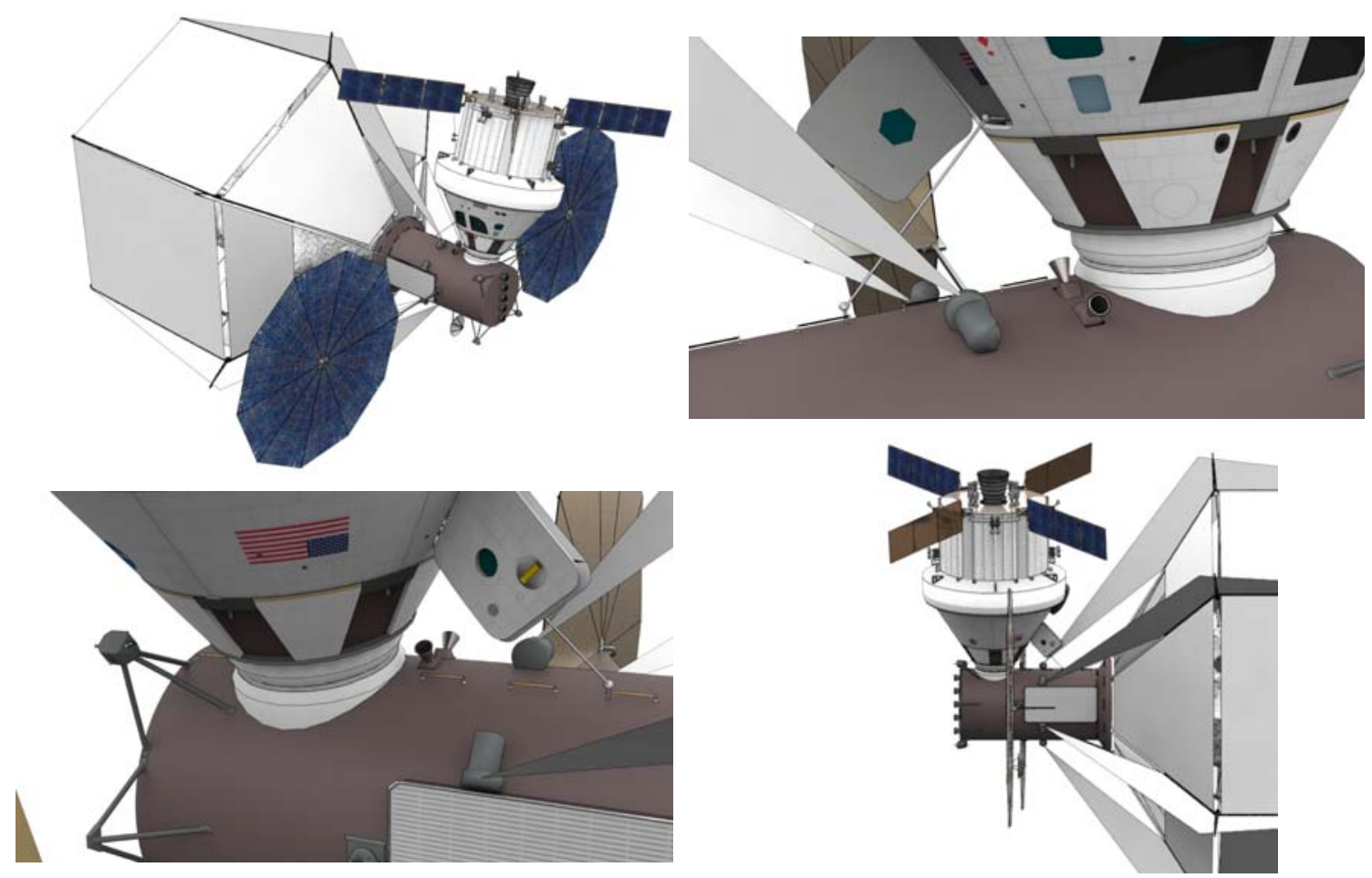

Figure 3 Radial Docking Analysis

\section{E. Relative Navigation Sensor Kit}

Orion Rendezvous, Proximity Operations and Docking (RPOD) with the ARV will require several navigation sensors and components whether it is performed autonomously or with pilot in the loop. This section describes the sensors and components required to successfully rendezvous and dock. Figure 2 shows the operational range for each sensor, along with a graphical depiction of their associated field of view (where applicable).

Two star trackers and S-band RF communications equipment are part of Orion's baseline to support nominal operations in Low-Earth Orbit (LEO) and beyond. The star trackers will be supporting long-range and mid-range bearing computation to a target vehicle, in addition to providing attitude updates to the Orion absolute navigation software. The S-band will provide range and range-rate measurements from the Orion to the ARV from 100km until the LOCI is the primary relative navigation sensor (approximately five to six kilometers).

Orion's Relative Navigation Kit, pictured in Figure 3, is comprised of two (2) Vision Navigation Sensors (VNS) and two (2) docking cameras into a single unit called the Laser Optical Camera Instrument (LOCI). LOCI is a single chassis, dual one-fault tolerant sensor suite. LOCI is mounted to the docking hatch, operating through the docking hatch window. The crew installs and connects LOCI prior to performing any rendezvous and docking maneuvers at least one day prior to rendezvous operations.

A prototype system, called Sensor Test for Orion RelNav Risk Mitigation (STORRM), consisting of a VNS and a docking camera, was tested on STS-134. The prototype system was demonstrated during docking, undocking and re-rendezvous operations. 


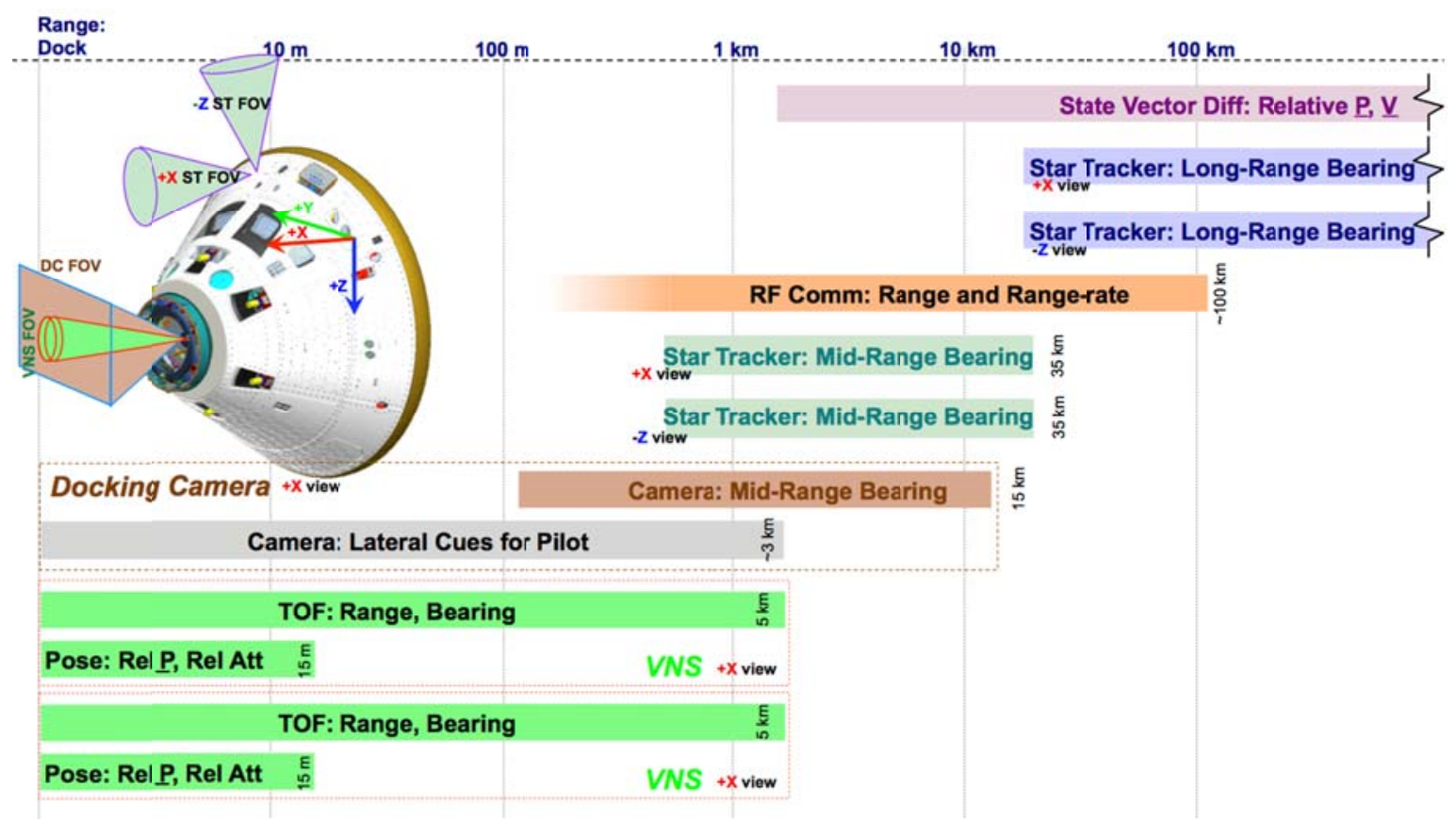

Figure 2: Orion Relative Navigation Sensor Operational Regime

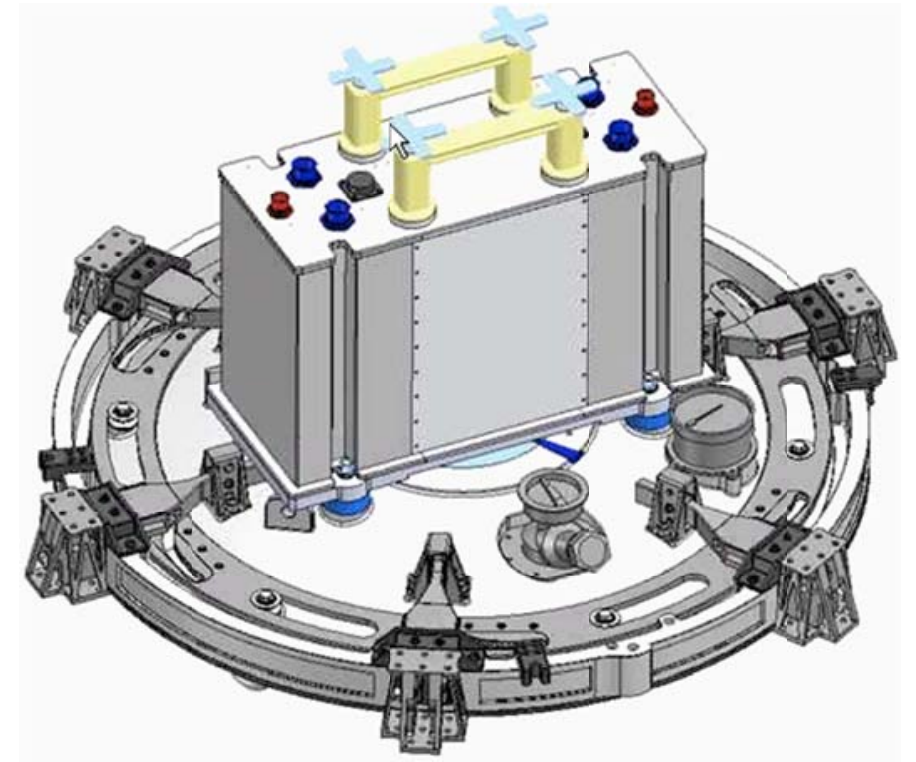

Figure 3: LOCI mounted to Orion docking hatch (interior view)

\section{F. Consumable Analysis}

For this feasibility study, the SM is assumed to fly with the full consumables capacity of $89.4 \mathrm{~kg}$ oxygen, $35.8 \mathrm{~kg}$ nitrogen, and 227.8kg water. Orion EM-2 carries sufficient ECLSS consumables to support 4 crewmembers for 18 nominal days and 3 contingency days. Additional capacity includes sufficient consumables to supply one contingency event. For this analysis, many cases were studied to determine the worst case O2, N2, and water 
storage required on the SM and CM. Consistent with the EM-2 baseline, the Orion cannot support all of the contingencies in the same mission, but it can accommodate any one of them. The list includes:

- An MMOD strike or other leak, followed by "Feed-the-Leak" for suit donning, and an unpressurized return to Earth

- A cabin depressurization and repressurization (after atmosphere contamination)

- Partial cabin depressurization and repressurization (after a failed hatch leak check)

- A contingency EVA using umbilicals, including depressurization and repressurization

- Engine out case which increases mission duration

- These cases, and the associated analysis, apply to the ARCM concept of operations with one exception. The duration of an unpressurized return to Earth or increased mission duration would change.

Consumables are driven by crew needs or by Orion contingencies. Orion Mission Functionality kits are used to provide oxygen during the EVA and to repressurize the vehicle. Therefore, consumables specifically related to ARCM activities are not drivers of consumable requirements. Figure 4 illustrates the allocation of each consumable to the mission requirements.

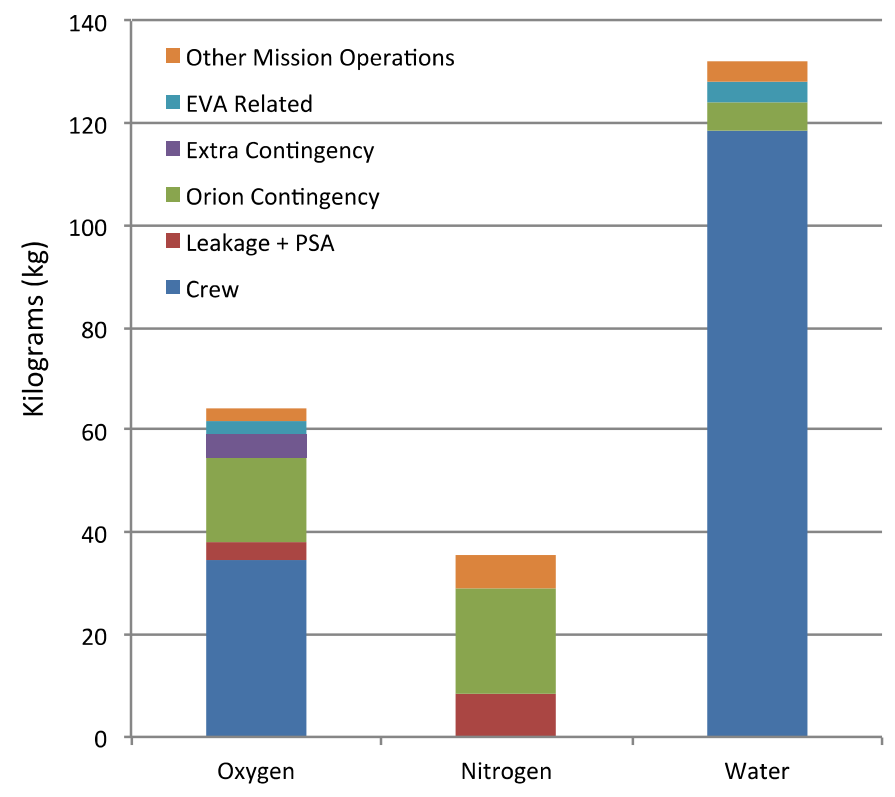

Figure 4 Consumables Required

\section{III.F.1.10xygen}

Oxygen is consumed by the crew and lost as a result of leakage or venting of several systems. The rate of metabolic consumption of oxygen varies depending on crew activity. The nominal 18-day Orion mission includes 11 exercise days and 7 non-exercise days. Leakage rates are affected by vehicle pressure. Oxygen is lost by venting the ullage volume of the amine swingbed system that controls $\mathrm{CO} 2$ and humidity, and may be lost if urine venting is included. When EVAs are performed, the oxygen (and other gases) in the cabin is vented so that the hatch can be opened. The EVA Repress Kit, not by the Orion tanks, replaces cabin gases. The PLSS provides oxygen for the EVA, some air will be lost to suit leakage. Orion oxygen will be used while the crew is on umbilicals during EVA preparation; this operation includes an oxygen purge to de-nitrogenate the spacesuit prior to cabin depressurization.

Contingency analysis for this mission has not identified any unique failure cases for the ARCM mission beyond those documented for Orion EM-2. However, due to the trajectories, if an unrecoverable depressurization occurs 
early in the mission, in which the crew has to don their suits in an unpressurized cabin, the total duration of suited time may be increased. The spacesuits have a considerably higher leak rate than the vehicle cabin, therefore the SM tanks must provide the additional oxygen needed.

Consumable management of oxygen for the mission is feasible because the total additional oxygen required for the extended duration, EVA operations, and additional contingency capacity is less than the quantity saved by the crew size reduction. Figure 5 depicts Orion oxygen consumption.

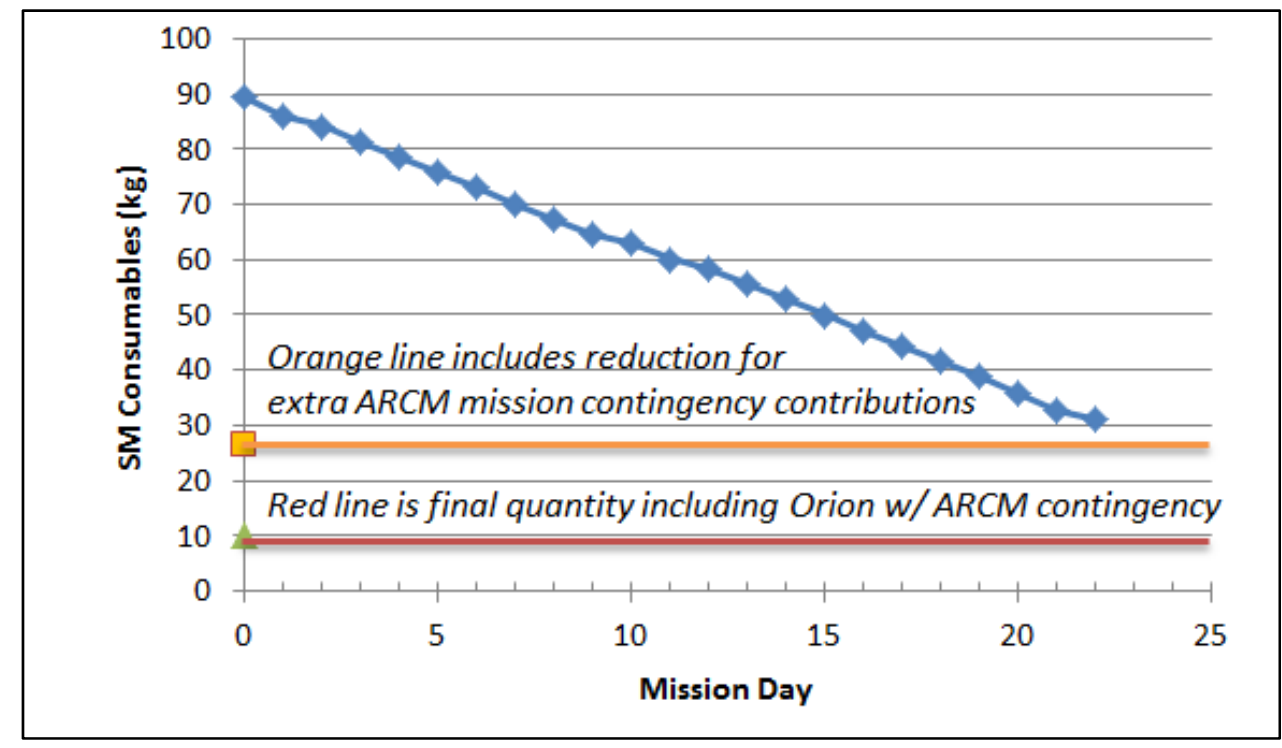

Figure 5: Orion SM Oxygen Usage

\section{III.F.1.2Nitrogen}

Nitrogen is provided for leakage and system venting makeup, as well as for repressurization from 10.2psia to 14.7psia before earth re-entry. Mission duration, system venting, and pressurization of a volume drive nitrogen use; it is not consumed metabolically. Nitrogen used to repressurize the cabin after EVA is provided by the Cabin Repress Kit, not the Orion SM tanks. Contingency nitrogen stores could be used to feed a leak while the crew dons their suits. Orion contingency stores sized for the "Feed the Leak" case must be preserved for this mission. The ARCM Concept of Operations does not change the nitrogen related contingency stores, and nitrogen use is not driven by crew size. The increase in the duration of the mission will increase nitrogen usage somewhat, but only by a very small amount. Figure 6 illustrates nitrogen consumption during the ARCM mission. 


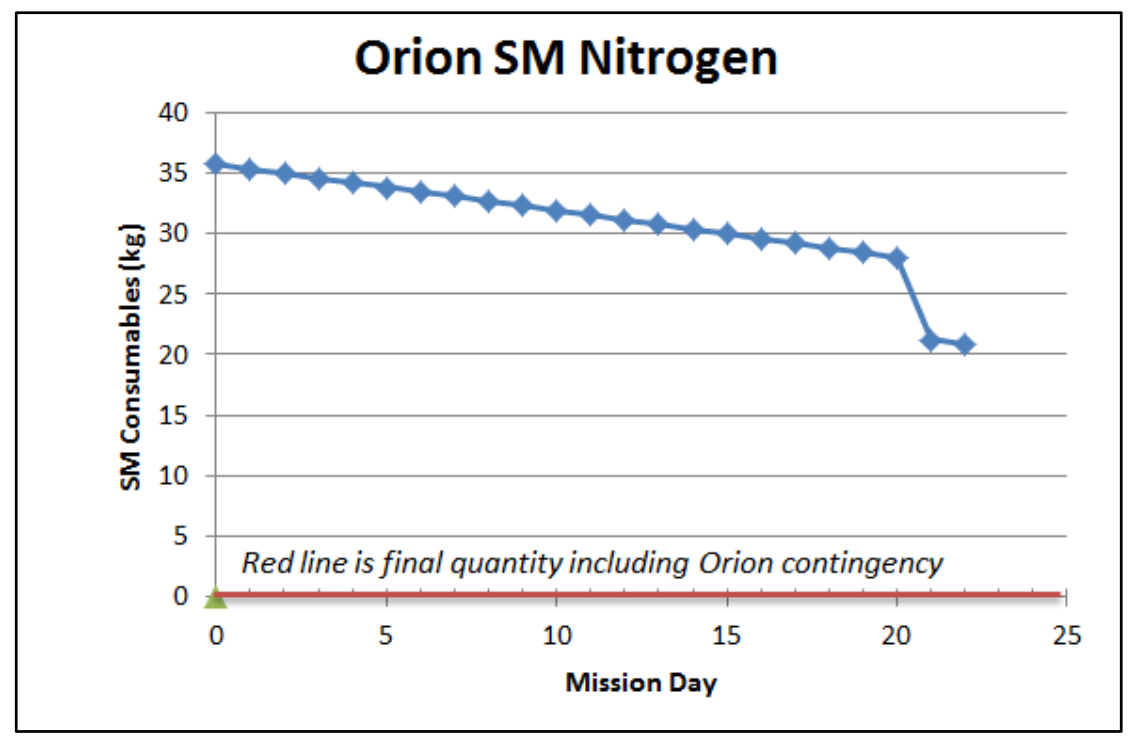

Figure 6: Orion SM Nitrogen Usage

\section{III.F.1.3Water}

Orion water use includes single-use and daily-use volumes. Single-use volumes include eyewash contingencies and mission events, such as rehydration prior to landing. Daily nominal use rates include drinking water and food rehydration water. The crew requires additional drinking water on EVA days, an addition to the ARCM mission scope as compared to Orion EM-2. The reduction in crew size from four to two substantially compensates for the additional water required for EVA hydration and the small increase in mission duration. Figure 7 shows water consumption throughout the mission.

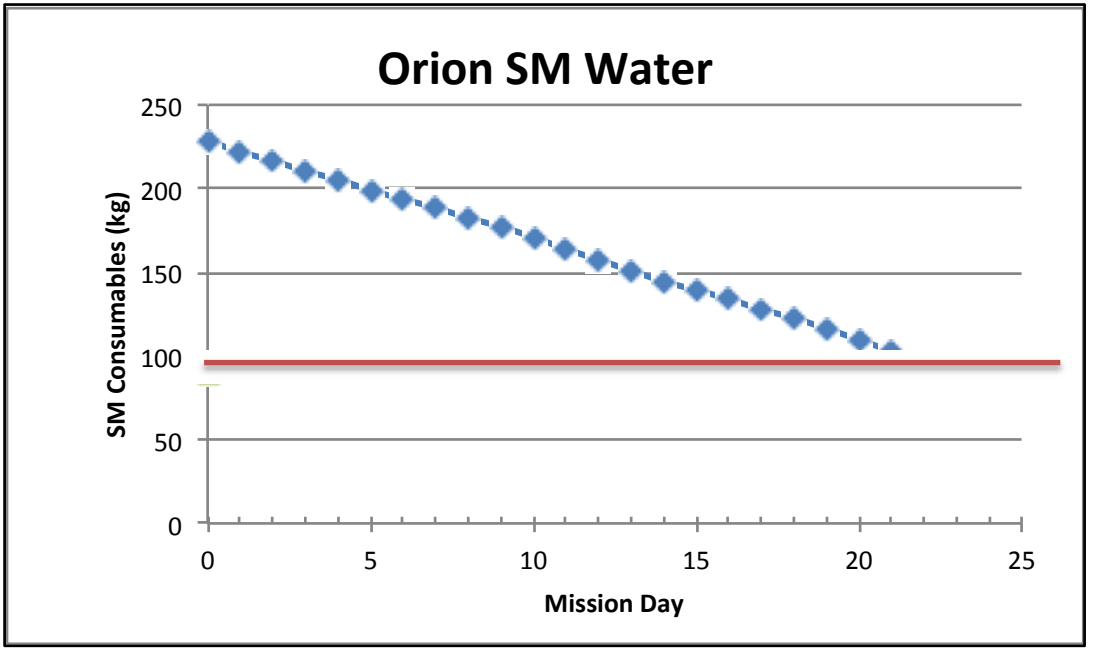

Figure 7: Orion SM Water Usage

\section{G. Integrated Orion ARCM Mass}

The ARCM mission kit masses were derived from a bottoms-up estimation of the required hardware. With the exception of the Relative Navigation Kit, all hardware masses were based on existing hardware elements and represent an accurate estimate of the additional mass required. The Relative Navigation Kit mass is derived from previous engineering estimates and represents the current best estimate. The EVA hardware kit mass of $283 \mathrm{~kg}$ 
includes the EVA Suit kit, PLSS, EVA Tools and EVA Communications mass estimates. The mounting hardware mass estimate is a parametrically derived mass to account for adapter plates required to stow the kit hardware in Orion.

Despite offloading crew and equipment, the ARCM mission required mass exceeds the Orion Gross Lift-Off Mass by $519 \mathrm{~kg}$. However the integrated trajectory analysis shows sufficient Orion Service Module $\Delta \mathrm{V}$ performance exists to execute the mission including performing part of the Trans Lunar Injection (TLI) burn.

Table 4: Orion ARCM Mass Delta

\begin{tabular}{|c|c|c|c|}
\hline & Orion Launch Mass for ARUM & Ib & kg \\
\hline \multirow{7}{*}{ 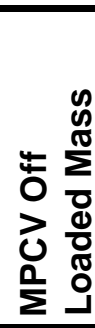 } & Crew & -360.0 & -163.3 \\
\hline & Crew Seat & -130.1 & -59.0 \\
\hline & GFE Crew Systems & -102.0 & -46.2 \\
\hline & Suits & -142.3 & -64.6 \\
\hline & SM Water* & 0.0 & 0.0 \\
\hline & SM Oxygen* & 0.0 & 0.0 \\
\hline & SM Nitrogen ${ }^{\star \star}$ & 0.0 & 0.0 \\
\hline \multirow{7}{*}{ 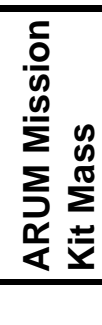 } & Cabin Repress Kit (26.5\% O2) & 189.5 & 85.9 \\
\hline & EVA Suit and Tools Kit & 624.8 & 283.4 \\
\hline & Sample Return Container & 22.0 & 10.0 \\
\hline & Docking Mechanism Kit & 751.8 & 341.0 \\
\hline & LAS Separation Hardware & 193.3 & 87.7 \\
\hline & Relative Navigation Kit & 97.0 & 44.0 \\
\hline & ARUM Mission Crew Module Mass Delta & 1144.1 & 519.0 \\
\hline
\end{tabular}

In addition to the Orion launch control mass, the feasibility analysis must also address the nominal and abort landed mass for Orion which is the load of the vehicle mass on the parachutes during landing. Table 5 shows the mass for nominal and abort landed mass.

The Service Module and docking mechanism are jettisoned prior to Earth entry and are therefore not included in the landed mass estimates. For the nominal landing, crew consumables and EVA repress air have been expended further reducing the landed mass. Following a successful mission, some of the EVA tools from the Orion EVA Tool Kit will likely remain in the ARV toolbox to support possible extensibility missions and up to $20 \mathrm{~kg}$ of asteroid samples will return in the sample container. The nominal landed mass is higher than the EM-2 baseline configuration by $39.9 \mathrm{~kg}$ and requires additional detailed analysis to identify mitigation options.

During an abort landing, this study assumes that the crew will not have had an opportunity to use any of the mission consumables and the landed mass in an abort scenario exceeds the EM-2 baseline value by even more than the nominal landed mass. Both the abort and nominal landed mass challenges must be resolved in the context of the greater Orion mass challenge where mitigations include accepting additional risk, increasing program dispersion parameters or reduced mission functionality. Further analysis is required. 
Table 5: Orion Nominal and Abort Landed Mass Delta

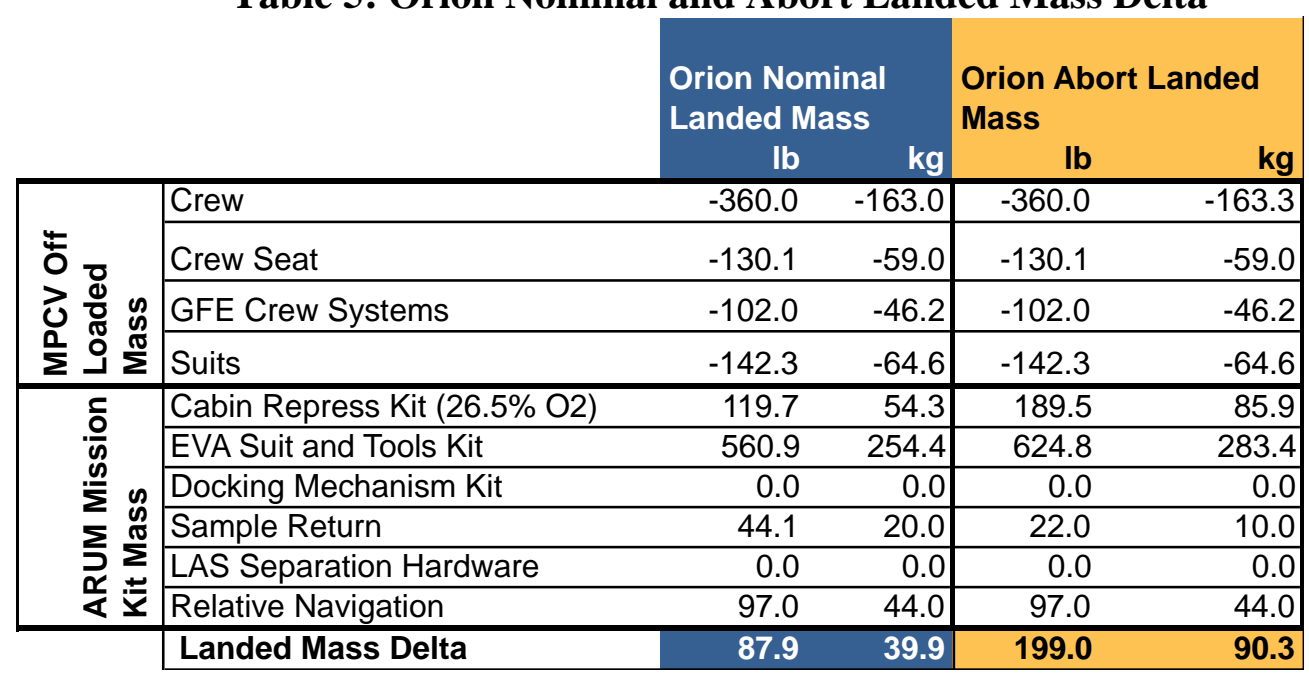

\section{CONCLUSION}

Mission kits are utilized to augment Orion with the necessary functionality without introducing costly new requirements to the mature Orion spacecraft design effort. Understanding how the mission kits are incorporated while minimizing cost, schedule and mass impacts will be is important on any spaceflight. The Asteroid Redirect Crewed Mission provides an exciting early mission for the Orion and SLS while providing a stepping-stone to even more ambitious missions in the future by leveraging the ARCM kit investments to provide the added functionality that will be beneficial to future exploration missions.

\section{ACKNOWLEDGMENTS}

The authors would like to acknowledge the support of the Asteroid Redirect Mission teams from JPL, LaRC, JSC, and KSC for their inputs. 